\title{
Characteristic Points Detection in ECG by Fusion of DP Matching and Neural Network
}

\author{
Non-member Sajjad Mohsin \\ (Muroran Institute of Technology) \\ Non-member Yukihisa Kurimoto \\ Member Yukinori Suzuki \\ (Muroran Institute of Technology) \\ Non-member Junji Maeda \\ (Muroran Institute of Technology) \\ (Muroran Institute of Technology)
}

\begin{abstract}
We propose a method for detecting characteristic points (CPs), the Q and S point, in an electrocardiogram. The method is a fusion of two methods, DP-matching and ART2 neural network. A multichannel ART neural network (MART) was used for the fusion. The method was tested using an MIT/BIH arrhythmia database. The rate of accuracy within $6 \mathrm{~ms}$ error was $99.6 \%$ for $\mathrm{S}$ point detection and $96.4 \%$ for $\mathrm{Q}$ point detection, indicating that the method is reliable.
\end{abstract}

Keywords: Electrocardiogram (ECG), CPs detection, DP-Matching, Neural Network, Fusion

\section{Introduction}

An electrocardiogram (ECG) is a record of the electrical potential induced by heart beats (Fig. 1). Since recording an ECG is a simple, noninvasive, and low cost procedure, it is widely used to diagnose heart diseases. Recent developments in telecommunication and information technology has enabled expert-based health care to be provided to at home ${ }^{(1)}$. An essential component for a system that enables expert-based health care to be provided to patients at home is a reliable ECG analyzer.

The most commonly used ECG analyzer is a beat-tobeat analyzer that detects QRS complexes in the ECG and diagnoses rhythm irregularity. The ECG analyzer can also diagnose an ischemia episode and myocardial infarction. For such diagnose, an ST-segment, which is the part of the ECG from the $J$ point to the onset of the $T$ wave, must be detected. Thus, the reliability of the ECG analyzer depends on how precisely it can detect the characteristic points (CPs), i.e., onset and/or offset of the waves and complexes constituting the ECG. There are two main problems that make detection of CPs difficult: 1) noises contaminating the ECG and 2 ) individual variation of waves and complexes in the ECG.

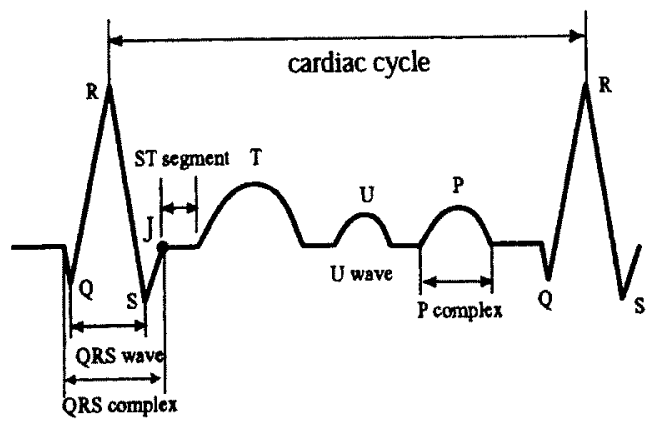

Fig. 1. The waves and complexes of an electrocardiogram.
The QRS wave is the most remarkable pattern in the ECG and is therefore used as a reference pattern for detecting the other waves and complexes. Thus, accurate detection of the QRS wave is a starting point in the development of a reliable ECG analyzer. This wave is detected by detection of two CPs, the $Q$ and the $S$ points. In this paper, we propose a method for detecting these two CPs, which could overcome the current problems in detection of CPs. We use DP-matching for overcoming the problem of noise contamination and a neural network of ART2 for overcoming the problem of individual pattern variation. These two methods are fused using a multichannel ART-based neural network (MART).

\section{CPs detection}

In this section, detection of CPs using ART2 and DP matching is described. Then the fusion of two methods using the MART is described. ECGs in the MIT/BIH arrhythmia database were used to test the reliability of our method. The ECGs have been digitized at a rate of $360 \mathrm{~Hz}$, a sampling rate that is not sufficient for to precise detection of CPs. We therefore transformed the ECGs into analog signals using Fourier and inverse Fourier transforms and then sampled them at a rate of $500 \mathrm{~Hz}$. This was performed using MATLAB.

2.1 Detection of CPs using the ART2 and DP matching ART2 is a self-organizing neural network that consists of attentional and orienting subsystems. The attentional subsystem is composed of an F1 layer of a feature detector, an F2 layer of a category representation, and bottom-up and top-down long-term memories (LTMs). Upward and downward connections between nodes in the F1 and F2 layers form a bottom-up and a top-down LTMs, respectively. ART2 recognizes input patterns through hypothesis testing cycle.

For detection of CPs using the ART2, we assume that the part of the ECG from the R point to the $Q$ point or $\mathrm{S}$ point can be approximated by a right-angled triangle. 
Ve stored 50 right-angled triangle patterns with differnt lengths of base in both the bottom-up and top-down TMs. These triangles are used as the initial values for attern recognition. For detection of the $Q$ point, the sart of the ECG $100 \mathrm{~ms}$ in length from the R point tovards the $P$ wave ( $Q R$ part) is the input to the $F 1$ layer. IRT2 recalls the right-angled triangle that is closest to he input pattern from the top-down LTMs. Then the eft end of the triangle locates the $Q$ point. The $S$ point $s$ also located in the same manner.

For detection of CPs using DP matching, we prepared ;wo template patterns that were empirically established sy observing a large number of QRS waves. For detecion of the $\mathrm{Q}$ point, we calculate distances between the $\mathrm{QR}$ part and the two template patterns. The template pattern showing a smaller distance is employed to detect the $Q$ point, which is stretched and/or shrunk by the algorithm of DP matching. This is symbolized as $\tilde{Q R}$. The $\mathrm{Q}$ point is detected in $\overline{Q R}$ based on a slope detection technique. This is found heuristically through trial and error. A sample $\tilde{Q R}(s)$ is chosen in the $\tilde{Q R}$ part. We compute $\Delta=\tilde{Q R}(s+n+1)-\tilde{Q R}(s+n)$ for $(n=1,2, \ldots, 10)$. If the number of $\Delta \geq 0$ is greater than or equal to seven, $\tilde{Q R}(s)$ is detected as the $\mathrm{Q}$ point. The first $\tilde{Q R}(s)$ is set at the left end of $\tilde{Q R}(s)$. The $\mathrm{S}$ point is also located in the same manner.

2.2 Fusion of ART2 and DP matching methods by MART MART is a multichannel neural network. Each channel consists of ART2. The architecture of MART is described in detail in reference ${ }^{(2)}$. We used MART with two channels to fuse the two methods, i.e., ART2 and DP matching. For detection of the $Q$ point, we prepared two input patterns. One is the QR part in the ECG, which is input to channel one of MART. Then nodes in the F2 layer of ART2 send signals to the F3 layer. The signal amplitude of an individual node is proportion to the activation level of each node in the $\mathrm{F} 2$ layer.

The other pattern is prepared as follows: From the QR part, we detect the $Q$ point by DP matching described in section 2.1. We make a rectangle using that $Q$ point. The base of the rectangle extends from the $R$ point to the $Q$ point. This rectangle is the input to channel two of MART. In this time, 50 rectangles are stored in both bottom-up and top down LTMs of ART2 of channel two beforehand. One stored rectangle is associated by the input pattern. Then nodes in the F2 layer send signals to the F3 layer. In this manner, nodes in the F3 layer receive signals from both channel one and channel two, so that the two methods, ART2 and DP matching, are fused by MART. The winner node in the F3 layer classifies the set of these patterns to a specific category. At that time, left end of the associated rectangle locates the $\mathrm{Q}$ point. The $\mathrm{S}$ point is also detected in the same manner. After the CPs have been detected, stored patterns in the LTMs of ART2 of channel one are updated by the learning equations of MART. In this manner, the stored patterns are modified by obtaining new information of the input pattern. For channel two, no learning is carried out.
Table 1. Standard deviations of $S_{\text {error }}$

\begin{tabular}{lcr} 
SD & Q point & S point \\
\hline Present method & 3.2 & 1.8 \\
ART & 3.8 & 2.9 \\
DP matching & 4.9 & 2.4 \\
\hline
\end{tabular}

\section{Results and Discussion}

The reliability of the present method for detection of CPs in an ECG was tested using ECGs stored in an MIT/BIH arrhythmia database. The record numbers of the ECGs used were 100, 103, 112, 113, 115, 117, 122,123 and 124. The CPs on these ECGs were first located visually. A total of 1,800 cardiac cycles of ECGs were used for evaluation. For evaluation of our method, the $Q$ point was defined as the first visually remarkable inflecting part of the ECG from the R point towards the $\mathrm{P}$ point. The $\mathrm{S}$ point was also defined as the first visually remarkable inflecting part of the ECG from the $\mathrm{R}$ point towards the $\mathrm{T}$ point. Detection errors were computed as $S_{\text {error }}=\left|n_{c}-n_{v}\right|$, where $n_{c}$ is the point on the ECG where a CP had been detected using the present method, and $n_{v}$ is the point on the ECG where a $\mathrm{CP}$ had been located visually. To evaluate the detected CPs, a part of the ECG including the QRS wave was expanded and drawn on the display. A referee visually determined the locations of the CPs.

The rates of accuracy with $S_{\text {error }} \leq 6.0 \mathrm{~ms}$ were $99.6 \%$ for $\mathrm{S}$ point detection and $96.4 \%$ for the $\mathrm{Q}$ point detection. Table I shows the standards deviation (SDs) of $S_{\text {error }}$ for the present method and for ART2 and DP matching. The SDs of the present method is lower than for detection of both $\mathrm{Q}$ and $\mathrm{S}$ points. The limit of SDs determined by the CSE committee for detection of onset of a $\mathrm{Q}$ wave and end of a QRS complex are 6.5 and 11.6 , respectively ${ }^{(3)}$. However, these limits are determined by data obtained from the CSE ECG library, which are different from ECG data obtained from the MIT/BIH arrhythmia database. It can be consider that we can evaluate the present method by the SDs for the detection error determined by the CSE committee, because ECGs in the MIT/BIH database are abnormal, which could be provide severe condition for the evaluation. The SDs are 3.2 and 1.8 for detection of $\mathrm{Q}$ and $S$ points detection, respectively, in our method, which SDs are well within the limits determined by the CSE committee. The experimental results show that the present method has good potential for detecting CPs on an ECG.

(Manuscript received Oct. 12, 2001)

\section{References}

(1) J. Lin, "From the guest editor: Current development in telemedicine", IEEE Engineering in Medicine and Biology, Vol. 18 , no. 4,1999

(2) Fernandez-Delgado, S.B. Amenerio, "MART: a multichannel ART-based neural network", IEEE Trans Neural Networks, vol. 9, no. 1, pp.139-149, 1998.

(3) The CSE working party, "Recommendation for measurement of standards in quantitative electrocardiography", European Heart Journal, vol. 6, pp. 815-825, 1985. 